\title{
Author Correction: Risk Factors of Cyberbullying Perpetration among School-Aged Children across 41 Countries: a Perspective of Routine Activity Theory
}

Qingyi Li ${ }^{1} \cdot$ Yan Luo $^{1} \cdot$ Zhichao Hao $^{1} \cdot$ Brenda Smith $^{2} \cdot$ Yuqi Guo ${ }^{3} \cdot$ Tyrone Cheng $^{4}$

Published online: 11 July 2020

(C) Springer Nature Switzerland AG 2020

\section{Author Correction: International Journal of Bullying Prevention}

https://doi.org/10.1007/s42380-020-00071-6

The original version of this article unfortunately contained a mistake in the author group section. The correct name of the second author is "Tyrone Cheng."

The online version of the original article can be found at https://doi.org/ 10.1007/s42380-020-00071-6

Qingyi Li

qli31@crimson.ua.edu

Yan Luo

yluo30@ crimson.ua.edu

Zhichao Hao

zhao6@crimson.ua.edu

Brenda Smith

bsmith2@sw.ua.edu

Yuqi Guo

yguo16@uncc.edu
Tyrone Cheng

tcheng6@kennesaw.edu

1 School of Social Work, University of Alabama, Room 104, Farrah Hall, 324 University Blvd, Tuscaloosa, AL 35487, USA

2 School of Social Work, University of Alabama, Little Hall 1030, Box 870314, Tuscaloosa, AL 35487, USA

3 School of Social Work, University of North Carolina at Charlotte, Charlotte, NC 28223, USA

4 Department of Social Work and Human Services, Kennesaw State University, Prillaman Hall, 3203, Kennesaw, GA 30144, USA 